\title{
Sensado de Espectro con Matrix Completion IZMA-SD para Redes Radio Cognitiva ${ }^{1}$
}

\section{Spectrum Sensing with Matrix Completion IZMA-SD for Cognitive Radio Networks}

\author{
O.F. Erazo, V.F. Miramá y J. E. Mora \\ Recibido: julio 27 de 2019 - Aceptado: septiembre 30 de 2019
}

\begin{abstract}
Resumen - Debido al crecimiento de las redes inalámbricas, se hace necesario un uso eficiente del espectro, una solución es Radio Cognitivo. En una de las etapas de esta tecnología se realiza el sensado de espectro, es decir determinar en una frecuencia si existen usuarios primarios y en caso de no existir, ocupar el espectro disponible; esto se logra al aplicar técnicas de sensado, cada técnica requiere de recursos hardware y puede identificar diferentes características de una señal. Hoy en día se utilizan altas frecuencias de propagación, es necesario que la etapa de procesamiento realice un muestreo Sub Nyquist, es decir a menos del doble de la máxima frecuencia, una alternativa de solución es utilizar un algoritmo basado en Matrix Completion, denominado por los autores como IZMA-SD. Los resultados muestran que en diferentes señales muestreadas al \%75 de Nyquist y bajo diferentes SNR, al aplicar el algoritmo se realiza la reconstrucción de la señal, a la cual se puede aplicar las técnicas de sensado.

Palabras clave-descomposición de valores singulares, desviación estándar, matriz de zona de interés de aproximación, matrix completion, norma nuclear, Radio coginitiva, sensado de espectro, sub-Nyquist.
\end{abstract}

Abstract - Due to the growth of wireless networks, efficient use of the spectrum is necessary, a solution is Cognitive Radio. In one of the stages of this technology, spectrum sensing is carried out, that is, determining on a frequency whether there are primary users and, if they do not exist, occupying the available spectrum;

${ }^{1}$ Producto derivado del proyecto de investigación "Sensado de espectro en banda ancha basado en matrix completion para redes de radio cognitiva".

O. F. Erazo, Universidad del Cauca, Cauca, Colombia, e-mail: olger@unicauca.edu.co.

V. F. Miramá, Universidad del Cauca, Cauca, Colombia, e-mail: vmirama@unicauca.edu.co.

J. E. Mora, Universidad del Cauca, Cauca, Colombia, e-mail: jorgemora@unicauca.edu.co.

Como citar este artículo: Erazo, O. F., Miramá, V. F., and Mora, J. E. Sensado de Espectro con Matrix Completion IZMA-SD para Redes Radio Cognitiva, Entre Ciencia e Ingeniería, vol. 13, no. 26, pp. 74-84, julio-diciembre 2019. DOI: https://doi.org/10.31908/19098367.1165.

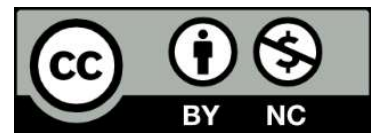

Attribution-NonCommercial 4.0 Intenational (CC By-NC 4.0) this is achieved by applying sensing techniques, each technique requires hardware resources and can identify different characteristics of a signal. Nowadays, high propagation frequencies are used, it is necessary for the processing stage to perform a Sub Nyquist sampling, that is, less than twice the maximum frequency. An alternative solution is to use an algorithm based on Matrix Completion, called by the authors like IZMA-SD. The results show that in different signals sampled at\% 75 of Nyquist and under different SNR, when the algorithm is applied, the reconstruction of the signal is performed, to which the sensing techniques can be applied.

Keywords - Cognitive radio, Interest zone matrix approximation, matrix completion, nuclear norm, spectrum sensing, singular value decomposition, Standard deviation, subNyquist.

\section{INTRODUCCIÓN}

$\mathrm{D}$ EBIDO al crecimiento de las redes inalámbricas y al uso del espectro, se hace necesario el uso eficiente de este recurso tan escaso, una solución es Radio Cognitivo (CR, Cognitive Radio); esta tecnología aprende de su entorno y una de las etapas de CR le corresponde al sensado de espectro (SS, Sensing Spectrum), es decir a evaluar su entorno, determinar si existen usuarios primarios (PU, Primary User) y en caso de no existir, ocupar el espectro disponible.

Cuando se desea trabajar solo con una frecuencia específica aparecen las técnicas en banda estrecha, entre ellas encontramos la detección de energía, la detección de características ciclo estacionarias, los filtros adaptativos, las redes neuronales, valores propios, la lógica difusa y técnica hibridas. Cada técnica requiere de recursos hardware y puede identificar diferentes características de una señal, esto se refleja en la probabilidad de detección, la cual cambia con respecto a la relación señal a ruido (SNR, Signal to Noise Ratio), las muestras a procesar cumplen con teorema de Nyquist, es decir que se requieren mínimo el doble de la máxima frecuencia.

El avance en la tecnología da paso al sensado de espectro en banda ancha (SSW, Sensing Spectrun Wideband), es decir a evaluar un rango de frecuencias y que junto a las altas frecuencias de propagación, hace necesario que los circuitos integrados de procesamiento puedan trabajar a menos del doble de la máxima frecuencia, es decir, se requiere de un muestreo 
Sub Nyquist. En este caso existen diferentes técnicas, una de ellas Matrix Completion, donde los datos perdidos son aquellas que no se muestrean y su objetivo es reconstruir la señal; para lograrlo se modifica un algoritmo que, a partir de la descomposición de valores singulares y la norma nuclear, se asignan en los datos perdidos valores semillas partir de la desviación estándar (SD, Standard Deviation), para así reducir el número de operaciones matemáticas y completar la matriz en un menor tiempo.

Una vez se reconstruye la señal, se aplica una de las técnicas de SS en banda estrecha para determinar la presencia de PU. Con el algoritmo planteado se requieren tres cuartos de las muestras empleadas por Nyquist, es decir que, si en Nyquist se requiere de 4 muestras, en el algoritmo modificado se necesitan 3 muestras, en pocas palabras no es necesario muestrear al doble de la máxima frecuencia, más bien se requiere muestrear a 1.5 veces la máxima frecuencia.

\section{SENSADO DE ESPECTRO EN RADIO COGNITIVE}

En los últimos años el incremento de usuarios con equipos móviles y las redes inalámbricas en las que estos se apoyan, han superado el tráfico de las redes cableadas [1], de acuerdo a un estudio de CISCO para el año 2015 Wi-Fi y los dispositivos móviles generan el $72 \%$ del tráfico en Internet, mientras que el $28 \%$ lo generan las redes fijas; para el año 2020 se espera que el $78 \%$ del tráfico lo generen las redes inalámbricas y solo un $22 \%$ las redes fijas, esto implica una crisis de espectro. En consecuencia existen políticas internacionales para realizar un uso eficiente del recurso de radio frecuencia (RF, Radio Frequency), por ejemplo en los Estados Unidos, partir del año 2010 se han definido planes[2] para hacer disponibles $500 \mathrm{MHz}$ de espectro que posibiliten el desarrollo de tecnologías emergentes en telecomunicaciones que impacten de forma positiva en la economía del país, con esta directriz la FCC (U.S. Federal Communications Commission) [3], empresas dedicadas a la asesoría para la administración del espectro[4] y universidades norteamericanas[5], han diseñado políticas para compartir el espectro[12], al igual que metodologías para la medición y análisis del mismo; los estudios demuestran que en algunos casos parte del espectro es muy utilizado o infrautilizado, por ejemplo la FCC en el año 2009 identifica que la banda de los $88-108 \mathrm{MHz}$ se encuentra generalmente ocupada, al igual que en $2.4 \mathrm{GHz}$, mientras que la banda comprendida entre los 1400 a $1850 \mathrm{Mhz}$ se presenta un bajo porcentaje de uso; por otra parte existen variaciones de uso temporal y geográfico, presentando huecos, es decir, disponibilidad de frecuencia, en bandas de hasta $2 \mathrm{MHZ}$ en determinados lapsos de tiempo.

Ante la creciente cantidad de usuarios de redes inalámbricas, la aparición de servicios que requieren mayores de ancho de banda y gran parte de las bandas de frecuencia licenciadas, se propone el concepto de Radio cognitiva (CR, Cognitive Radio), tecnología que busca dar solución a este problema haciendo un uso eficiente de los recursos espectrales que se encuentren disponibles. Un sistema CR se caracteriza por la capacidad cognitiva y la auto-reconfiguración, por ello debe ser flexible, sensar espectro, aprender y adaptarse al entorno con el que interactúa, elementos que se desprenden de la definición dada por Mitola [6-7] y Haykin [8]; en esta tecnología se identifican dos elementos, los usuarios primarios( $\mathrm{PU}$, Primary User) y los usuarios secundarios (SU, Secondary User), los primeros quienes han licenciado parte del espectro para su uso y los segundos, no licenciados, quienes verifican el espectro disponible y lo emplean, siempre que no se interfiera con los PU.

El avance de CR fue posible por la adición de capacidades a los dispositivos de radio definidos por software SDR (Software Defined Radio) [7-12], prestaciones como: sensar, aprender, adaptarse y usar espectro; un SDR es un equipo de radio, reprogramable y reconfigurable, estos dispositivos modifican de forma dinámica parámetros de operación como la frecuencia, la potencia y la modulación, logrando hasta el momento trabajar con un rango de frecuencias de hasta $5 \mathrm{GHz}$ y anchos de banda de $100 \mathrm{MHz}$. La limitación en el rango de frecuencia se debe a la arquitectura de procesamiento basados en Arreglos de compuertas programables en campo (FPGA, fieldprogrammable gate array) y en el desempeño de los convertidores análogo a digital (ADC, analog-to-digital converter) que trabajan a $400 \mathrm{Ms} / \mathrm{s}$ con resolución de 16 bits [11], por ello se requiere de algoritmos que sensen, administren, compartan y permitan la movilidad del espectro, aprovechando los recursos existentes a nivel de hardware.

La CR para lograr la detección, el uso eficiente del espectro y minimizar la interferencia con PU, ha desarrollado diferentes técnicas de sensado de espectro (SS, Spectrum Sensing), identificando espacios negros, grises y blancos [8]. Los espacios negros representan bandas que permanecen ocupadas gran parte del tiempo, presentando una alta potencia de interferencia; los espacios grises hacen referencia a bandas que se encuentran parcialmente ocupados, presentando una baja potencia de interferencia; los espacios blancos corresponden a bandas libres de interferencia. El desempeño del SS se mide generalmente por métricas como la probabilidad de detección

$\left(P_{D}\right)$, probabilidad de falsa alarma $\left(P_{F A}\right)$ y probabilidad de la detección fallida $\left(P_{M D}\right)$.

Las técnicas SS se clasifican bajo diferentes criterios, entre ellas se tiene: detección basada en el Rx (cooperativos), el sensado basado en interferencia y detección basada en el transmisor (locales) [13-14]. Aunque también se pueden clasificar como Coherentes y no Coherentes, en la primera se conocen las características de la señal y en la segunda no se conocen las características de la señal; las técnicas igualmente se pueden clasificar basados en el ancho de banda, en banda estrecha y banda ancha [15-16]. En cuanto al número de muestras de la señal se pueden clasificar en sensado Nyquist y Sub-Nyquist [17].

El sensado Nyquist y Sub-Nyquist [17] es otra forma de clasificar las técnicas SS, la primera clasificación agrupa a las técnicas que se basan en un conjunto de observaciones de una señal, dadas por un ADC muestreando a la tasa de Nyquist, en la cual la frecuencia de muestreo debe ser superior al doble de la frecuencia de la señal a evaluar, por ello si se trabaja con altas frecuencias se presenta un mayor tiempo de procesamiento, un incremento de los recursos de hardware y mayor complejidad computacional. El SS Sub-Nyquist busca encontrar el número mínimo de mediciones para reconstruir una señal y determinar 
la disponibilidad de determinada frecuencia, existiendo límites y desafíos como el tamaño de la matriz de sensado, la implementación en hardware, la incertidumbre ante el ruido y la recuperación de la señal [18].

Desde un análisis matemático en el sensado no compresivo, muestreo Nyquist, se parte de la prueba de una hipótesis binaria que indica si está libre u ocupado un canal en particular, el modelo hipotético de la detección de un transmisor por parte de un SU se puede describir cómo se ilustra en la ecuación 1 [18].

$$
y(k)= \begin{cases}H_{0}: & n(k) \\ H_{1}: & s(k)+n(k)\end{cases}
$$

Donde $y(k)$ corresponde a la muestra a ser analizada en un instante de tiempo $k, n(k)$ representa el ruido y s $(k)$ es la señal del PU.

\section{PROBLEMA}

Independiente de la técnica empleada en SS o el detector usado, los errores son inevitables debido al ruido aditivo, el número limitado de muestras y la aleatoriedad de los datos observados, hecho que conlleva a dos tipos de error. El primer tipo de error involucra la falsa alarma que ocurre cuando un canal que está libre se detecta como ocupado y el segundo tipo de error se presenta cuando existe una detección fallida que hace referencia a que un canal ocupado es detectado como libre. El primer error implica que no se pueda ocupar el canal libre y el segundo que un SU interfiera a un PU. Por ello el desempeño de un detector se caracteriza por dos parámetros, la probabilidad de detección fallida $\left(P_{M D}\right)$ y la probabilidad de falsa alarma $\left(P_{F A}\right)$, las cuales se definen en la ecuación 2.

$$
\begin{aligned}
& P_{F A}=\operatorname{Prob}\left\{\text { Decidir } H_{1} \mid H_{0}\right\} ; \\
& P_{M D}=\operatorname{Prob}\left\{\text { Decidir } H_{0} \mid H_{1}\right\} ;
\end{aligned}
$$

En el sensado Sub-Nyquist o Nyquist Incompleto, se encuentran algunas técnicas [17], una de ellas se denomina sensado compresivo (CS, Compresive Sensing), en el cual la señal es adquirida y comprimida simultáneamente solo con información esencial, posterior a ello se recupera a partir de una pocas muestras, la tasa de muestreo es inferior a Nyquist, siendo una solución adecuada para reducir el tiempo de procesamiento, acelerar el proceso de escaneo y trabajar con frecuencias superiores a las del oscilador de la unidad de proceso del usuario CR.

En el sensado compresivo, muestreo sub-Nyquist, se propone detectar la presencia o ausencia de la señal PU desde un mínimo número de mediciones, por ello el modelo se puede reformular en términos del CS [18] como se ilustra en la ecuación 3.

$$
y=\left\{\begin{array}{l}
H_{0}: \quad \Omega n \\
H_{1}: \quad \Omega(s+n)
\end{array}\right.
$$

Donde $y$ corresponde a la medición compresiva que contiene solamente la información más significante para la detección, $n$ representa el ruido gaussiano, $\Omega$ es una matriz de sensado conocida de $\mathrm{M}$ filas por $\mathrm{N}$ columnas, $\mathrm{M}<\mathrm{N}$, y s es la señal del PU. Existe una modificación al CS, se denomina Completar
Matriz (MC, Matrix Completion), esta busca recuperar una matriz de bajo rango desde pocas muestras, para SS la señal recuperada no necesariamente debe ser exacta a la original, pero si mantener características que permitan identificar la presencia o ausencia de un PU en varias bandas [19].

En la próxima generación de redes celulares se requiere explotar las oportunidades de tiempo, frecuencia y espacio, por lo anterior se necesitan algoritmos locales eficientes en la detección de la señales en banda ancha, ante bajas SNR, que empleen un conjunto pequeño de muestras, exijan un bajo costo computacional y de implementación, para suplir además las necesidades generadas por las aplicaciones emergentes como la ultra banda ancha y las aplicaciones de radar en las cuales su muestreo superan las capacidades tecnológicas vigentes.

\section{SOLUCIÓN}

Nosotros usamos Matrix Completion, MC, es una modificación al sensado compresivo; Candes y otros [20] definen el $\mathrm{MC}$, como el proceso matemático que busca recuperar datos de una matriz desde pocas muestras.

El objetivo de recuperar los datos de una matriz $\mathrm{M}$ con $\mathrm{n} 1$ filas por $\mathrm{n} 2$ columnas, pero que solo se observan $\mathrm{m}$ muestras que representan las entradas, cuyo número es mucho menor que n1.n2 del total de las celdas de la matriz.

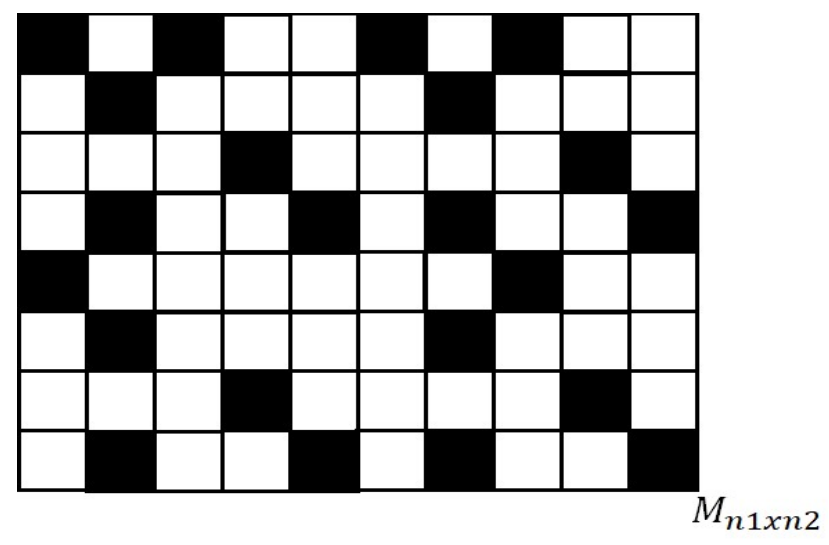

Fig. 1, Datos de la matriz a recuperar.

Se observa en la Fig. 1 que en la matriz M las celdas de color negro representan las muestras y las celdas de color blanco los datos a recuperar. Como requisito la matriz $\mathrm{M}$ no debe tener filas o columnas sin ninguna muestra. Además, la matriz debe ser de bajo rango; el rango de una matriz es el número máximo de columnas o filas respectivamente que son linealmente independientes, por ello que el rango por filas y el rango por columnas siempre son iguales; un bajo rango indica que la información es redundante, pero no todas las matrices se pueden recuperar, observe la siguiente matriz de rango 1:

$$
M=e_{1} e_{n}^{*}=\left[\begin{array}{ccccc}
0 & 0 & \ldots & 0 & 1 \\
0 & 0 & \ldots & 0 & 0 \\
\vdots & \vdots & \vdots & \vdots & \vdots \\
0 & 0 & \ldots & 0 & 0
\end{array}\right]
$$


El vector $e_{i}$ es un vector de base canónica en un espacio Euclidiano, donde todas las entradas son ceros, pero solo el enésimo es 1; este tipo de matriz no se puede recuperar ya que no se sabe si toda la matriz se conforma de ceros y además en diferentes técnicas de recuperación los datos no conocidos, las celdas vacías, se asignan con ceros.

Como una matriz $M$ de bajo rango se puede aproximar por medio de la descomposición de valores singulares (SVD, Singular Value Descomposition), Emmanuel Candes [20] plantea la solución para encontrar los datos perdidos a partir de una matriz de bajo rango genérica.

$$
M=\sum_{k=1}^{r} \sigma_{k} \mu_{k} v_{k}^{*}
$$

Donde $r$ es el rango, $\sigma_{k}$ es una vector que contiene los valores singulares por la izquierda de la matriz $M, \mu_{k}$ contiene los valores singulares de $M$ y $v_{k}^{*}$ es el vector de valores singulares por la derecha traspuesta. Normalmente se aplica SVD a una matriz de bajo rango que cuenta con todas las entradas, obteniendo información útil que permita reconstruir la matriz original y obtener matrices de rango inferior, pero parecidas a $M$. Por lo anterior como el número valores singulares $\mu_{k}$, más el número de vectores traspuesto por la derecha $v_{k}^{*} \mathrm{y}$ el número de vectores por la izquierda es menor a la cantidad de valores de la matriz $M$ completa, se utiliza esta técnica en la compresión de imágenes.

\section{A. Norma Nuclear y SVD.}

Existen diversos algoritmos para MC, uno de ellos se denomina Norma Nuclear y SVD. Un algoritmo de este tipo requiere contar con un número grande de muestras y además que estas se encuentren uniformemente distribuidas, con al menos una observación por fila y columna. El planteamiento inicial de Emmanuel Candes de una matriz supuesta de rango 1 de la forma $x y^{*}, x, y \in \mathbb{R}^{n}$ de manera que el $(i, j)$ enésimo entrada está dada por:

$$
M_{i, j}=x_{i} y_{i}
$$

Permitirá recuperar los datos de la matriz si se soluciona un problema de optimización:

$$
\begin{aligned}
& \text { minimizar } \quad \operatorname{rango}(X) \\
& \text { Sujeto } a X_{i j}=M_{i j}(i, j) \in \Omega
\end{aligned}
$$

Donde $\Omega$ es el conjunto de entradas, $X$ es la variable decisión y rango $(X)$ es igual al rango de la matriz $X$. Los algoritmos que se apliquen para llegar a una solución exacta requieren de mayor tiempo de procesamiento y es un problema NP-hard, ya que no tienen algoritmos polinomiales, por lo que un algoritmo para resolverlo puede tardar mucho tiempo.

La solución al problema de optimización se relaciona con la suma de los valores singulares sobre un conjunto de restricciones; la suma se llama la forma nuclear y está dada por la ecuación 8 .

$$
\|X\|_{*}=\sum_{k=1}^{n} \sigma_{k}(X)
$$

Donde $\sigma_{k}(X)$ denota el k-ésimo mayor valor singular de $X$. La optimización según Candes está dada por la ecuación 9.

$$
\begin{array}{lc}
\text { minimizar } & \|X\|_{*} \\
\text { Sujeto a } & X_{i j}=M_{i j}(i, j) \in \Omega
\end{array}
$$

\section{B. Algoritmo IZMA_SD}

Gil Shabat y Amir Averbutch [21] plantean un algoritmo para la aproximación de matriz (IZMA, Interest Zone Matrix Aproximattion); uno de los métodos propuestos por los autores plantea que una matriz de bajo rango se puede aproximar al minimizar la norma de Frobenius teniendo en cuenta las restricciones dadas en la norma nuclear de la matriz.

$$
\begin{gathered}
\text { minimizar } \quad\|\mathrm{P} X-\mathrm{P} M\|_{F} \\
\text { Sujeto a } f(X)>=0
\end{gathered}
$$

Donde $\|.\|_{F}$ es la norma de Frobenius, $P M$ es la proyección que indica la entradas que se desean aproximar de $M$, la proyección de $X$ es $\mathrm{P} X=B \odot X$, donde $B$ es la matriz de ceros y unos, ceros representan los datos perdidos; $\odot$ es una multiplicación puntual.

Sin embargo, al aplicar el algoritmo IZMA en una señal se encuentra que las matrices aproximadas requieren de muchos cálculos para encontrar los datos perdidos o que los datos recuperados presentan una gran diferencia si se conoce a priori su valor.

En Matrix Completion las entradas conocidas no deben cambiar de valor mientras se minimiza la función objetivo sujeta a las restricciones, ya que se desea minimizar el rango de la matriz. Los datos perdidos de la matriz son aquellas que no se muestrean y el objetivo es reconstruir la señal; en este trabajo se modifica un algoritmo IZMA de Shabat y Averbuch, descrito en [21], que busca minimizar la suma de valores singulares sujeto a un conjunto de restricciones; la modificación del mismo consiste en asignar a los datos perdidos valores semillas, partir de la desviación estándar (SD, Standard Deviation) de las muestras, este valor puede ser negativo o positivo, depende del valor anterior y posterior al dato perdido, a este algoritmo se llamará IZMA_SD.

Para su aplicación en CR, la etapa SS se propone un modelo general del sistema por medio de 4 bloques fundamentales, integrando a IZMA la SD, tal como se describe en la Fig. 2.

$\mathrm{Al}$ adicionar estos valores y reducir el número de iteraciones para encontrar la aproximación a la matriz, se busca reducir el tiempo de procesamiento $\mathrm{y}$ el número de operaciones matemáticas.

Los bloques finalmente se pueden describir mediante el algoritmo 1, SS basado en Matrix Completion IZMA_SD. 


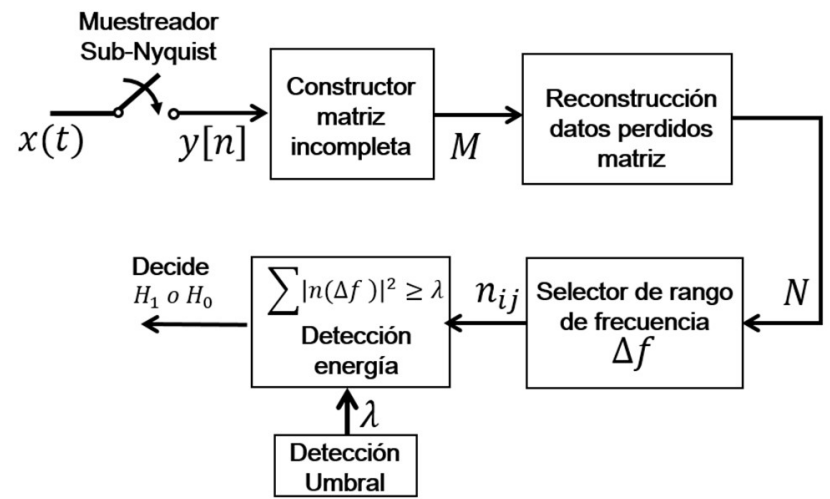

Fig. 2. Modelo general del SS IZMA_SD por bloques. Fuente: por autores.

Algoritmo 1. SS basado en Matrix Completion IZMA_SD [21]

\begin{tabular}{l} 
Entradas: \\
Mm Es un vector con muestras 1.75 veces la máxima \\
frecuencia \\
Salida: Decisión $-\mathrm{H}_{1} \mathrm{o} \mathrm{H}_{0}$. \\
\hline $\mathrm{l}$. $\leftarrow$
\end{tabular}

\section{1: $L \leftarrow$}

Constructor matriz incompleto a partir de $\mathrm{mm}$

2: $\quad N \leftarrow L \odot B$

3:

integre semilla desviación estandar desde matriz $N$

4: $\quad \lambda_{\min } \leftarrow 0 \quad \lambda \leftarrow$ infinito

5: $\quad \lambda_{\max } \leftarrow\|M\|_{*}$

6: Repetir:

7: $\lambda_{\text {prev }} \leftarrow \lambda$

8: $\lambda \leftarrow\left(\lambda_{\max }+\lambda_{\text {min }}\right) / 2$

9: $\quad X \leftarrow I Z M A-$

$S D$ aproxima $M \odot B$ para puntos sujetos a $\|X\|_{*} \leq \lambda$

10: $\quad$ error $\leftarrow\|\mathcal{P} X-\mathcal{P} M\|_{F}$

11: $\quad$ si error $\geq$ tol entonces

12: $\quad \lambda_{\min } \leftarrow \lambda$

13: $\operatorname{sino}$

14: $\quad \lambda_{\max } \leftarrow \lambda$

15: $f i n s i$

16: hasta $($ error $<$ tol $) y\left[\left(\lambda-\lambda_{\text {prev }}\right)<\lambda_{\text {tol }}\right]$

17: Selección del rango de frecuencia.

18: Aplicación de SS en banda estrecha a la matriz X.

19. Decisión $\leftarrow$ Estado del canal.

Constructor matriz incompleta. Este bloque se encarga de ejecutar dos procedimientos, el primero denominado "Constructor matriz incompleta a partir de $\mathrm{mm}$ " y el segundo se identifica como "integre semilla desviación estándar desde matriz N".

El procedimiento "Constructor matriz incompleta a partir de $\mathrm{mm}$ " toma un vector $\mathrm{mm}$ y adiciona ceros en los espacios entre muestra y muestra, los valores ceros son espacios a completar, posterior a ello a la matriz se adicionan 3 filas, las cuales son réplicas de la primera, la matriz resultante se conoce como $L$.

La siguiente etapa del procedimiento se encarga de multiplicar elemento a elemento la matriz $L$ por una matriz $B$, esta última se conforma de ceros y unos, esta contiene el mismo número de filas y columnas de la matriz $L$, organizada de tal manera que cada fila y columna se intercalan los ceros y unos, para evitar que existan filas o columnas con solo ceros, las filas impares inician con cero y las filas pares inician con 0 ; este orden es utilizado para completar posteriormente los ceros con la SD y la matriz resultante se denomina matriz $N$.

$\mathrm{Si}$ la señal se considera periódica, la función integre semilla desviación estandar, algoritmo 2, asigna a los espacios vacíos, un valor que corresponde a SD, el cual es positivo si el valor de la muestra anterior y siguiente es positivo, al igual que es positivo si uno de ellos también lo es y el otro es cero. Si el anterior y el siguiente son negativos se proceden a cargar la semilla con la SD pero negativa. Si uno es negativo y el otro es mayor igual a cero, se asigna al punto medio entre el próximo y anterior, al cual se le resta dos veces el SD y finalmente se divide entre dos.

\section{Algoritmo 2. Integre semilla desviación estándar}

\section{Entradas:}

$N \quad$ Matriz a insertar semilla

INICIO2 1 si es fila impar y 0 si fila es par

Salida: M Matriz con valores semilla $S D$.

$M \leftarrow \quad 1: S D \leftarrow$ Desviacion estandar de $N$

2: columnas $\leftarrow$ numero de comumnas de $N$

4: semilla $\leftarrow N$

5: si (fila=0) entonces

6: $\quad i \leftarrow 0$

7: de otro modo

8: $\quad i \leftarrow 1$

9: fin si

10: Repetir:

10: $\quad$ anterior $\leftarrow N(i-1)$

11: $\quad$ próximo $\leftarrow N(i+1)$

12: si anterior $>=0$ y próximo $>=0$ entonces

13: $\quad m(1, i) \leftarrow S D$

14: fin si

15: $\quad$ si anterior $<0$ y próximo $<0$ entonces

16: $\quad m(1, i) \leftarrow-S D$

17: fin $\mathrm{si}$

18: $\quad x y \leftarrow($ anterior $+\mid$ próximo $\mid)-2 * S D$

19: $\quad x y \leftarrow|x y| / 2$

20: si anterior $>=0$ y próximo $<0$ entonces

21: $\quad m(1, i) \leftarrow x y$

22: fin si

23: $\quad$ si anterior $>0$ y próximo $>=0$ entonces

24: $\quad m(1, i) \leftarrow-x y$

25: fin $s i$

26: $\quad i \leftarrow \mathrm{i}+2$

27: mientras $i<=$ columnas

28: retorne $m$ 


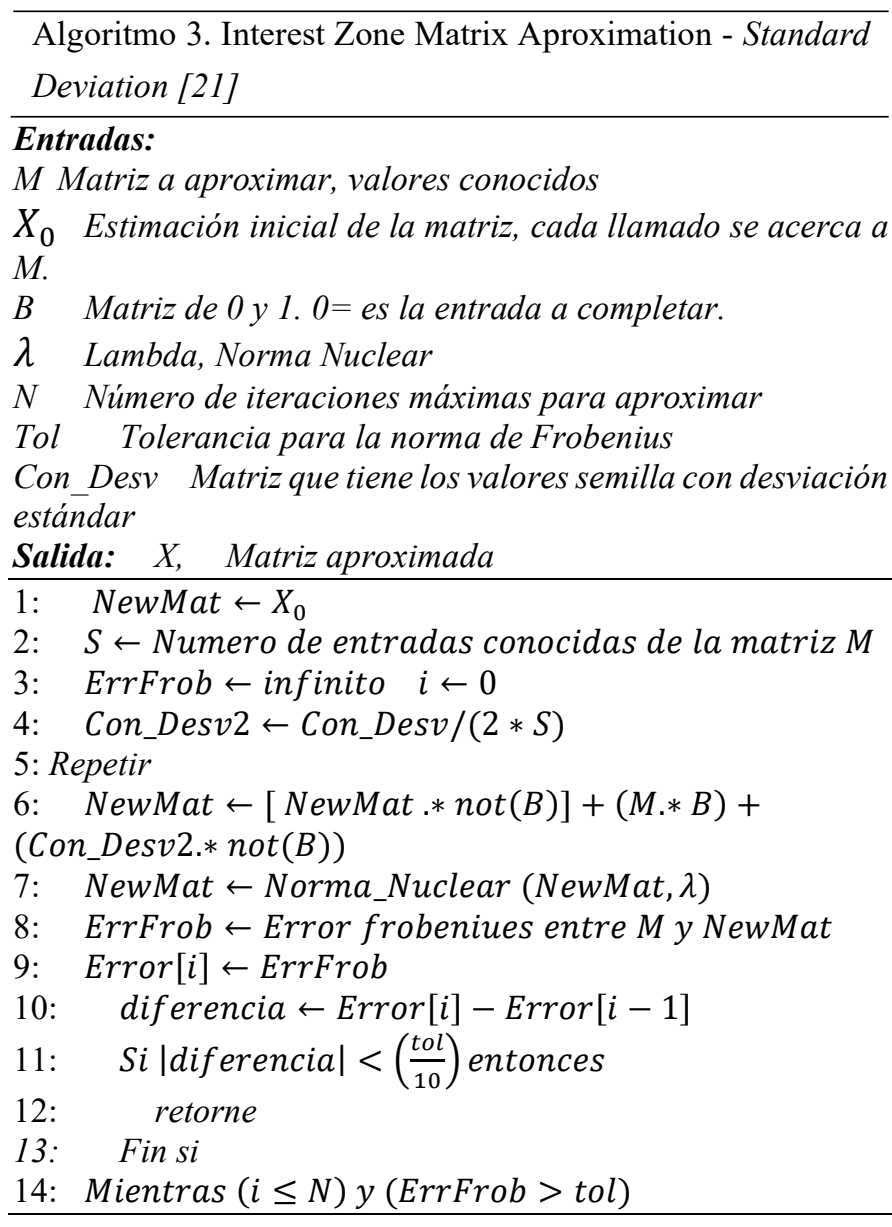

Reconstrucción de datos perdidos. El algoritmo IZMA_SD que aproxima la matriz $\mathrm{X}$ a partir de los valores semilla SD, requiere $\lambda$ para realizar los procesos de aproximación; con respecto a los autores [21] se adiciona en el algoritmo 3, paso 4, Con_Desv2 la cual es una matriz que cuenta con la SD semilla de la matriz $\mathrm{M}$ dividida entre dos veces el número de datos conocidos de la matriz M. También se encuentra otra modificación en el paso 6, al encontrar que NewMat es la suma de la matriz de valores aproximados de NewMat previa con los valores conocidos igual a cero, más las muestras conocidas de matriz a completar $\mathrm{M}$ y finalmente adiciona los valores que contiene los valores semillas de $\mathrm{M}$ en la matriz Con_Desv2.

Selector de rango de frecuencia. El procedimiento Selección del rango de frecuencia, permite seleccionar el rango a evaluar del total de los rangos de frecuencia muestreados y completados en la matriz X. Es decir que el resultado de este bloque es un vector que se envía al siguiente bloque.

Detector de energía. En esta etapa se ejecuta la aplicación de SS en banda estrecha a la matriz X, se aplica DE como técnica SS para detección de PU. En el algoritmo 4 se describe el procedimiento a desarrollar para detectar la presencia del PU, en especial el valor del umbral del ruido se determina a partir de la medición del entorno en un rango de frecuencias que se conoce no existen PU.
Algoritmo 4. Detección de Energía [22]

\section{Entradas:}

$X \quad$ Matriz a evaluar.

$\lambda$ Umbral del ruido

Salida: Estado_del_canal

1: Pxx $\leftarrow$ Periodograma, estimación del DSP.

2: Hpsd $\leftarrow$

Energía de la señal, distribución de potencia en el dom. de frec.

$3: i \leftarrow 0$

4: Repetir

5: $\quad$ sumatoria_rango $(i) \leftarrow$

sumatoria Pxx por conjunto de 16 frecuencias

6: $\quad i \leftarrow i+1$

7: Mientras $i<=32$

8: $\quad i \leftarrow 0 \quad$ contador $\leftarrow 0$

9: Repetir

10: $\quad$ si sumatoria_rango $(i)>\lambda$ entonces

11: $\quad$ contador $\leftarrow$ contador +1

12: fin si

13: Mientras $i<=32$

14: Si (contador $>2$ )

15: $\quad$ Estado_del_canal $\leftarrow 1$ "ocupado"

16: si no

17: $\quad$ Estado_del_canal $\leftarrow 0$ "libre"

18: Fin si

\section{RESULTADOS Y DISCUSIÓN}

\section{A. Validación de los códigos}

Los diagramas de flujo se codifican en Matlab y para validar MC IZMA_SD se asumen diferentes señales deterministas como seno, coseno, solo ruido, bpsk y qpsk, las cuales se generan a diferentes frecuencias y se muestrean con tasas inferiores a Nyquist.

Inicialmente se trabaja con una señal seno a $9 \mathrm{~Hz}$, la cual inicialmente se conforma de 24 muestras.

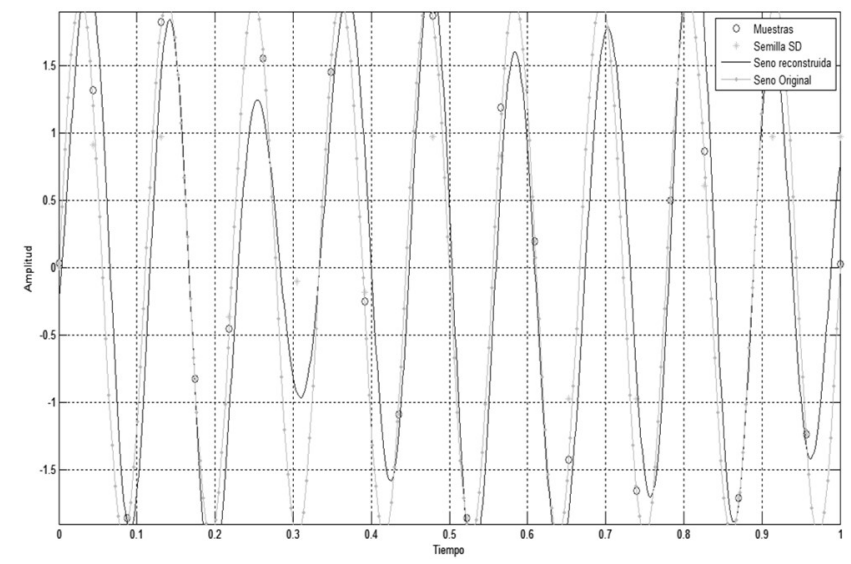

Fig. 3, Señal seno $9 \mathrm{~Hz}$, reconstruida.

A los datos muestreados se generan pérdidas, es decir que se asignan ceros en el $50 \%$ de las muestras, es decir que el algoritmo MC IZMA_SD se carga con 12 muestras conocidas; en el algoritmo 1, paso 2, se denomina matriz $N$. 
Una vez se ejecuta el algoritmo MC IZMA_SD se logra recuperar las muestras perdidas y se observa como algunas de ellas pueden tener menor amplitud, por ello en el algoritmo se plantea la sumatoria de las 4 primeras filas de la matriz resultante.

Finalmente, una vez se realiza la suma de la señal, se procede a graficar las muestras, la señal reconstruida y la señal original, tal como se observa en la Fig. 3.

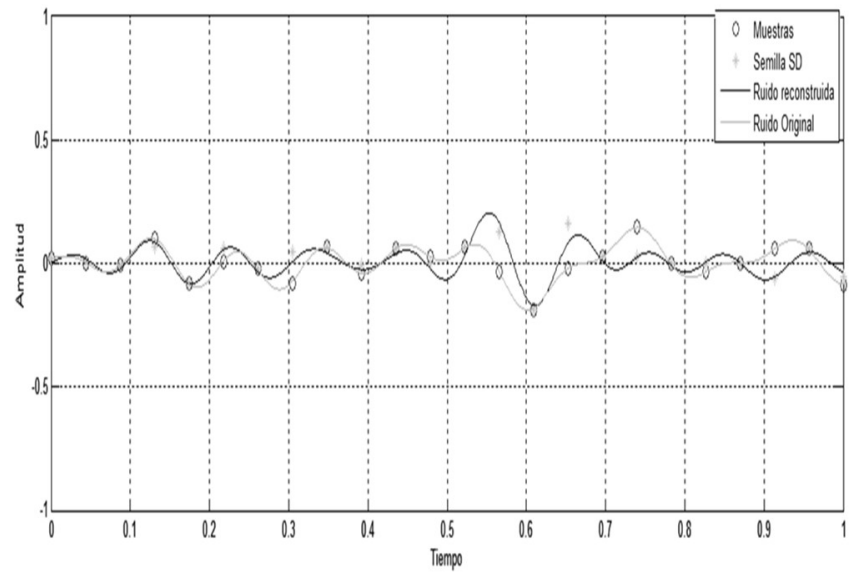

Fig. 4. Ruido, reconstruido a partir de 12 muestras.

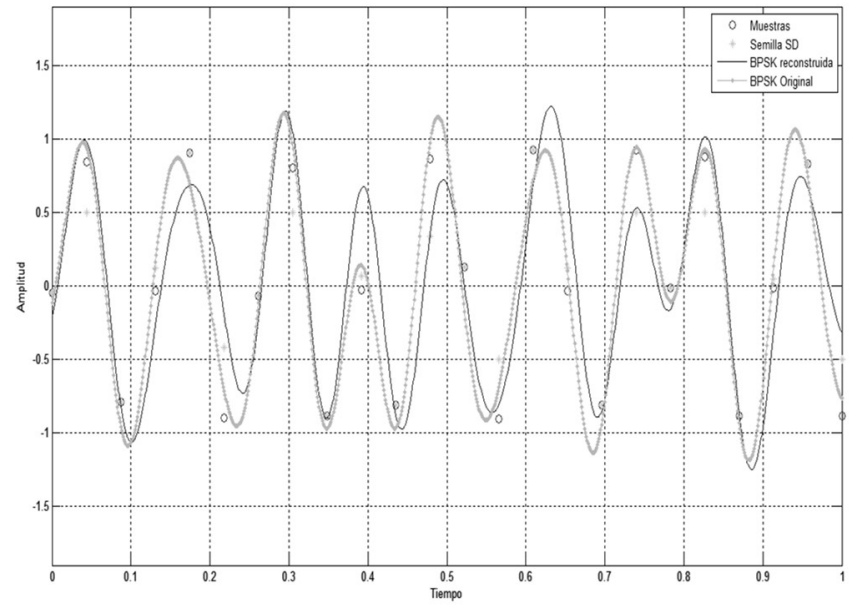

Fig. 5. Señal BPSK reconstruida a partir de 12 muestras.

Ya que existe la posibilidad que en una frecuencia a muestrear no exista usuario alguno, solo ruido, se aplica el algoritmo y se observa cómo se reconstruye ese tipo de señal.

Al aplicar el algoritmo IZMA_SD se logra reconstruir una señal BPSQ y QPSK, tal como se observa en la Fig. 5 y Fig. 6 respectivamente.

Las señales BPSK y QPSK reconstruidas se asemejan a la señal original y a estas muestras se puede aplicar la técnica SS en banda estrecha.

Sin aplicar el algoritmo IZMA_SD en una señal seno con una frecuencia de $9 \mathrm{~Hz}$ y 12 muestras, en la figura 7 se observa que la reconstrucción no es posible, el número de cálculos para reconstruir la matriz es igual a 10 y el error medio cuadrático es 0 , tanto la norma nuclear de la matriz corrupta o con datos perdidos es igual a la norma nuclear de la matriz restaurada.

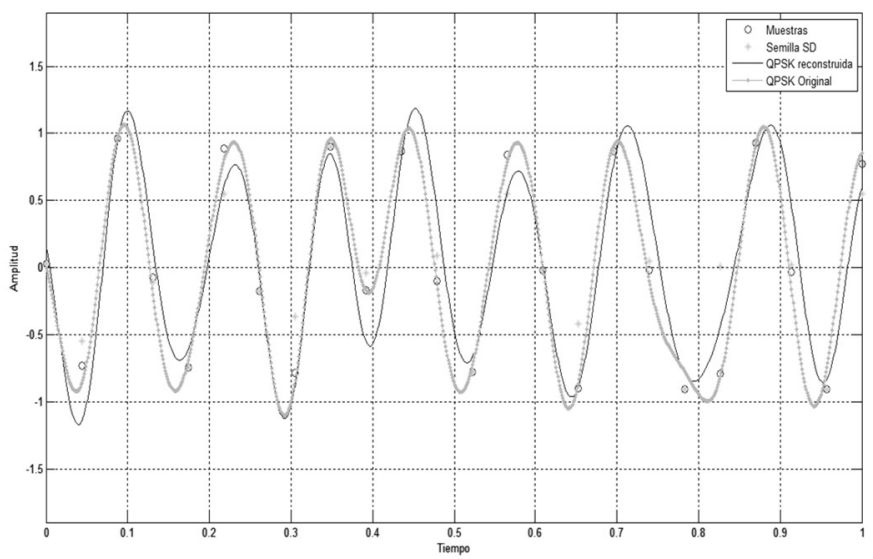

Fig. 6, Señal QPSK reconstruida a partir de 12 muestras.

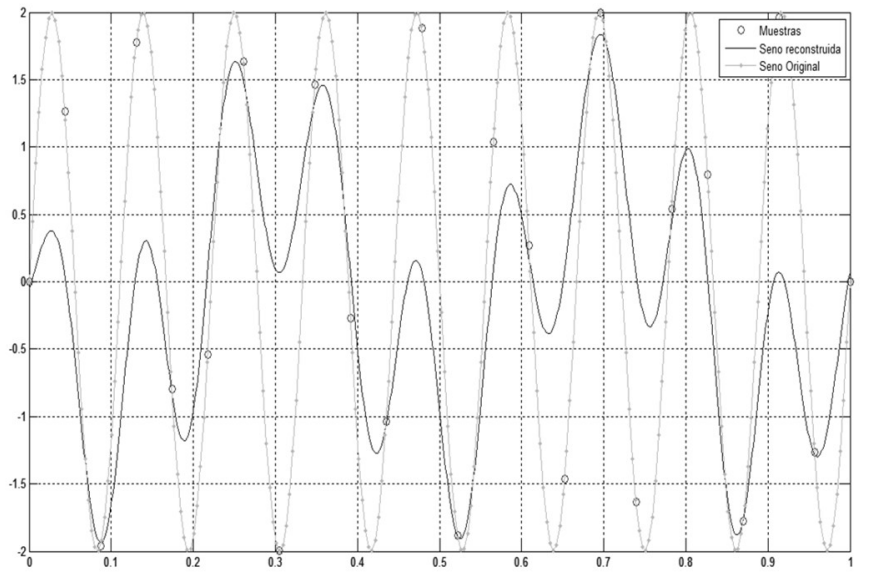

Fig. 7, Señal seno $9 \mathrm{~Hz}$ reconstruida sin MC IZMA_SD a partir de 12 muestras

\section{B. Resultados de simulación algoritmo reconstrucción de la señal}

Una vez se codifican los algoritmos, para validar la efectividad del algoritmo de reconstrucción MC IZMA_SD, se plantean escenarios con diferentes señales y diferentes SNR.

Se trabaja con 5 tipos de señales determinísticas como: bpsk, qpsk, seno, coseno y solo ruido, con una frecuencia de $9 \mathrm{~Hz}$ y con solo 12 muestras válidas; se realizan 12.000 simulaciones para reconstruir diferentes tipos de la señal y finalmente se cuenta con 24 muestras reconstruidas por simulación, ahora interesa determinar la diferencia de norma nuclear, error medio cuadrado, la cantidad de operaciones para recalcular la matriz recuperada y el grado de semejanza, esta última se determina por medio de la correlación cruzada.

Los resultados de cada simulación se registran en un archivo plano, el cual es decodificado por un software desarrollado en Visual Studio 2017 y almacenado en una base de datos, otro módulo de software se encarga de tabular los datos, para ello consulta desde la base de datos desarrollada en MySql los tipos de señala a evaluar y organiza estadísticas por grupos de rangos de SNR de $4 \mathrm{db}$.

La Fig. 8 muestra la diferencia entre la norma nuclear inicial de la matriz a recuperar y la norma nuclear final de la matriz recuperada es superior a 1 para SNR inferiores a $5 \mathrm{~dB}$; un valor de diferencia superior a 1 indica que la matriz recuperada no se aproxima. 


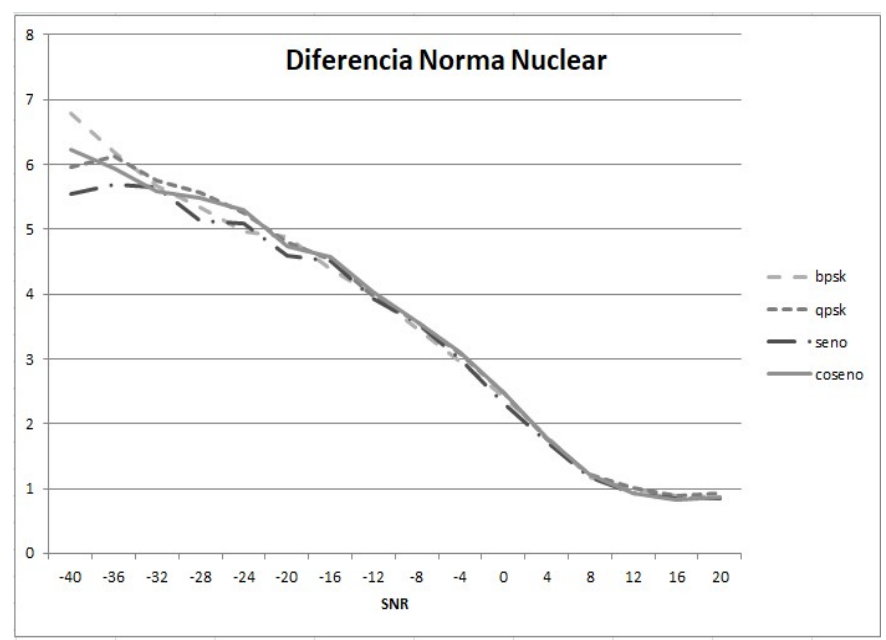

Fig. 8, Diferencia entre la norma nuclear inicial y la norma nuclear final de la señal recuperada vs SNR.

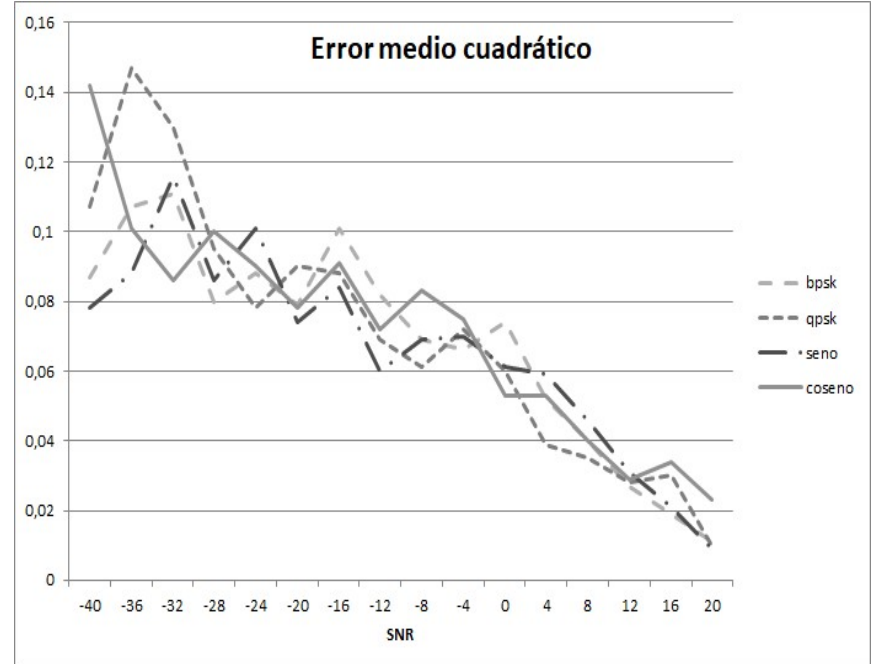

Fig. 9. Error medio cuadrático entre la señal original y la señal recuperada vs SNR. Fuente: por autores.

En la Fig. 9 se observa un comportamiento similar en el error medio cuadrático, la figura 4.3 demuestra que este es superior a 0.08 en SNR inferiores a $-10 \mathrm{~dB}$ y esta se reduce en SNR superiores a $2 \mathrm{~dB}$, nuevamente el algoritmo presenta un menor error en la aproximación de las muestras perdidas en buenas condiciones de canal.

Un aspecto importante en la ejecución del algoritmo es tiempo que tarda en generar la matriz aproximada, en la figura 10 se observa que se requieren más de 300 aproximaciones en SNR inferiores a $-8 \mathrm{~dB}$ y esta se reduce a 250 aproximaciones en SNR superiores a $8 \mathrm{~dB}$, nuevamente el algoritmo presenta mayor velocidad al recuperar muestras perdidas en buenas condiciones de canal.

El desempeño del algoritmo MC IZMA_SD a partir del número de aproximaciones expresada en centésimas de segundo depende del recurso hardware, en el cual además intervienen los módulos AD y el procesamiento de la señal.

El número de aproximaciones es un aspecto es importante en la reconstrucción de la señal, ya que, sin aplicar el SD, el algoritmo IZMA tan solo ejecutaba 10 aproximaciones, con un

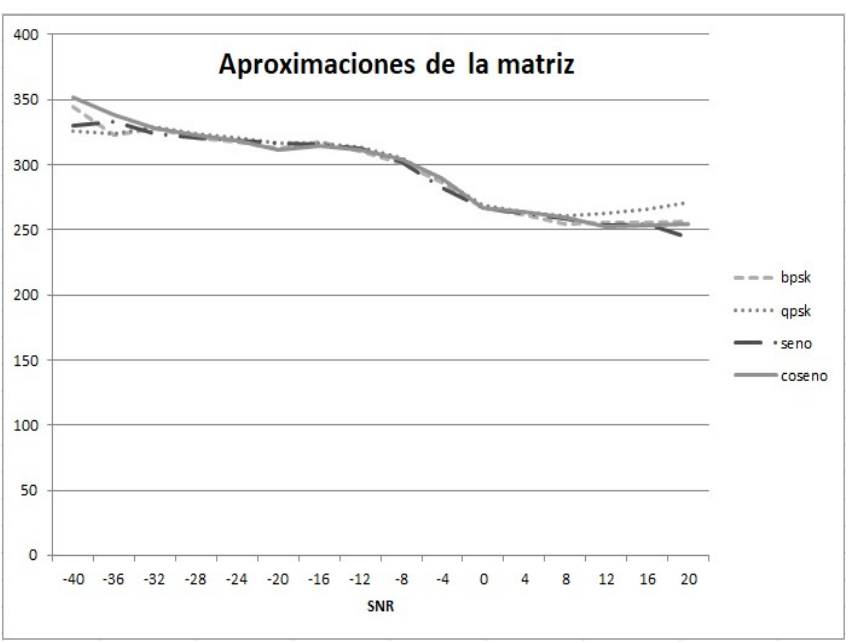

Fig. 10, Numero de aproximaciones necesarias para llegar a la señal recuperada vs SNR. Fuente: por autores.

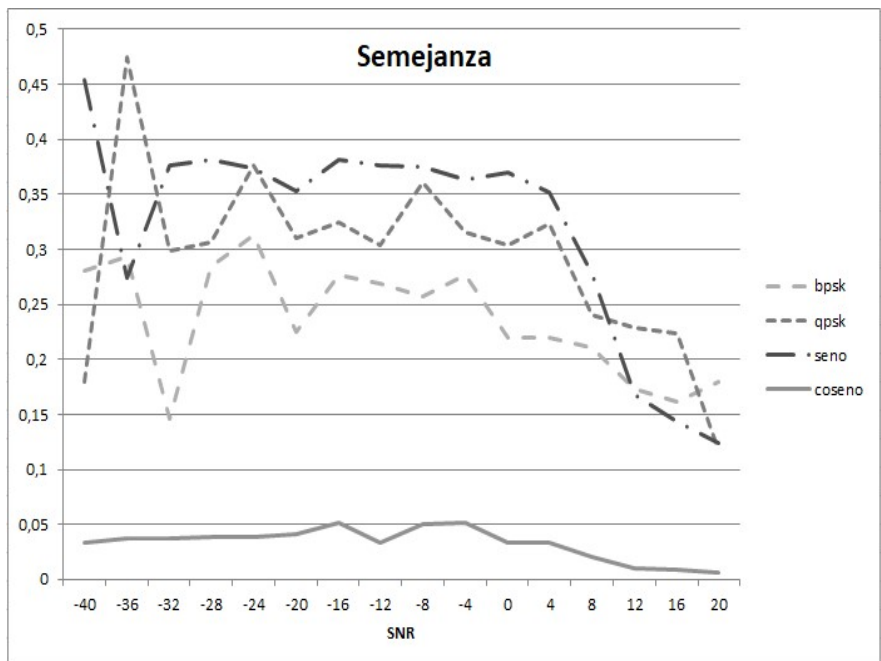

Fig. 11, Semejanza entre la señal de referencia y la señal recuperada vs SNR. Fuente: por autores.

error medio cuadrático cercano a cero y los datos recuperados se acercaban a cero, siendo imposible recuperar la señal.

El valor de semejanza de la señal, Fig. 11, es un indicador de similitud entre la señal original con todas sus muestras y la señal recuperada por medio del algoritmo IZMA-SD con el 50\% de las muestras conocidas.

La correlación cruzada indica que para los cuatro tipos de señal se observa mayor similitud si la señal tiene una SNR superior a $2 \mathrm{~dB}$. Cabe aclarar que un valor de cero significa similitud total y valores superiores a 0.4 diferencias entre la señal original y la recuperada.

La señal coseno tiene mayor similitud ya que en SNR superiores a $4 \mathrm{~dB}$ el error se aproxima a 0 , esto se debe a que al insertar valores semilla con SD este se acerca más al comportamiento de la señal coseno.

\section{Resultados de simulación al aplicar SS por detección de energía.}

Una vez se reconstruye la señal, el diagrama de flujo de SS DE previamente codificado en Matlab se ejecuta y se realizan 12.000 simulaciones empleando modulaciones BPSK y QPSK. Para sistematizar el proceso de estimación de $\mathrm{P}_{\mathrm{d}}$, la $\mathrm{P}_{\mathrm{fa}} \mathrm{y}$ la $\mathrm{P}_{\mathrm{md}}$, se construye un formulario en Visual Studio 2017, encargado 
de decodificar los resultados de las simulaciones generados en Matlab, se almacena los registros en una tabla de la base de datos diseñada en Mysql, se consulta los resultados en la base de datos bajo diferentes condiciones de canal y los tipos de modulación ya descritos.

TABLA I

PROBABILIDAD DE FALSA ALARMA CON DE

\begin{tabular}{|c|c|c|c|c|c|}
\hline $\begin{array}{l}\text { No. } \\
\text { Reg. }\end{array}$ & Muestras & $\begin{array}{c}\text { SNR } \\
\text { superior }\end{array}$ & $\begin{array}{c}\text { SNR } \\
\text { inferior }\end{array}$ & $\begin{array}{c}\text { Muestras } \\
\text { detectadas }\end{array}$ & $P_{f a}$ \\
\hline 1 & 113 & -12 & -14 & 113 & 1,00 \\
\hline 2 & 113 & -10 & -12 & 113 & 1,00 \\
\hline 3 & 113 & -8 & -10 & 113 & 1,00 \\
\hline 4 & 114 & -6 & -8 & 114 & 1,00 \\
\hline 5 & 114 & -4 & -6 & 114 & 1,00 \\
\hline 6 & 198 & -2 & -4 & 198 & 1,00 \\
\hline 7 & 177 & 0 & -2 & 177 & 1,00 \\
\hline 8 & 198 & 2 & 0 & 92 & 0,46 \\
\hline 9 & 201 & 4 & 2 & 15 & 0,07 \\
\hline 10 & 116 & 6 & 4 & 12 & 0,10 \\
\hline 11 & 116 & 8 & 6 & 10 & 0,09 \\
\hline 12 & 114 & 10 & 8 & 12 & 0,11 \\
\hline 13 & 114 & 12 & 10 & 0 & 0,00 \\
\hline 14 & 98 & 14 & 12 & 0 & 0,00 \\
\hline 15 & 92 & 16 & 14 & 0 & 0,00 \\
\hline
\end{tabular}

El software desarrollado por los autores cuenta con tres funciones, la primera permite administrar los elementos a consultar, la segunda consolida los resultados de las técnicas de detección bajo diferentes condiciones de canal; la tercera función permite graficar los resultados.

TABLA II

PROBABILIDAD DE DETECCIÓN CON DE

\begin{tabular}{|r|r|r|r|r|r|}
\hline $\begin{array}{r}\text { No. } \\
\text { Reg. }\end{array}$ & Muestras & $\begin{array}{c}\text { SNR } \\
\text { superior }\end{array}$ & $\begin{array}{c}\text { SNR } \\
\text { inferior }\end{array}$ & $\begin{array}{c}\text { Muestras } \\
\text { detectadas }\end{array}$ & $\mathrm{P}_{\mathrm{d}}$ \\
\hline 1 & 115 & -12 & -14 & 14 & 0,12 \\
\hline 2 & 113 & -10 & -12 & 22 & 0,19 \\
\hline 3 & 113 & -8 & -10 & 35 & 0,31 \\
\hline 4 & 114 & -6 & -8 & 43 & 0,38 \\
\hline 5 & 114 & -4 & -6 & 90 & 0,79 \\
\hline 6 & 201 & -2 & -4 & 170 & 0,85 \\
\hline 7 & 175 & 0 & -2 & 160 & 0,91 \\
\hline 8 & 198 & 2 & 0 & 187 & 0,94 \\
\hline 9 & 201 & 4 & 2 & 184 & 0,92 \\
\hline 10 & 120 & 6 & 4 & 108 & 0,90 \\
\hline 11 & 120 & 8 & 6 & 110 & 0,92 \\
\hline 12 & 113 & 10 & 8 & 108 & 0,96 \\
\hline 13 & 114 & 12 & 10 & 114 & 1,00 \\
\hline 14 & 97 & 14 & 12 & 97 & 1,00 \\
\hline 15 & 92 & 16 & 14 & 92 & 1,00 \\
\hline & & & & & \\
\hline
\end{tabular}

Una de las funciones del software desarrollado por los autores permite consolidar los datos representados en matrices, por ello la tabla I demuestra que el algoritmo detector de energía en SNR inferiores a $-2 \mathrm{~dB}$ presenta una probabilidad de falsa alarma cercana al $100 \%$. En este caso la técnica DE presenta un mal comportamiento en bajas SNR, es decir que detecta la señal $\mathrm{y}$ en realidad en el canal solo existe ruido.

En la tabla II se observa que la técnica de SS DE presenta una buena probabilidad de detección para SNR superiores a -4 $\mathrm{dB}$.

La tabla III demuestra que el algoritmo detector de energía en SNR inferiores a $-2 \mathrm{~dB}$ presenta una probabilidad de detección fallida superior al 26\%. En este caso la técnica DE presenta un mal comportamiento en bajas SNR, es decir que detecta que el canal esta libre y en realidad este se encuentra ocupado.

TABLA III

PROBABILIDAD DE DETECCIÓN FALLIDA CON DE

\begin{tabular}{|r|r|r|r|r|r|}
\hline $\begin{array}{r}\text { No. } \\
\text { Reg. }\end{array}$ & Muestras & $\begin{array}{c}\text { SNR } \\
\text { superior }\end{array}$ & $\begin{array}{c}\text { SNR } \\
\text { inferior }\end{array}$ & $\begin{array}{c}\text { Muestras } \\
\text { detectadas }\end{array}$ & $P_{\text {md }}$ \\
\hline 1 & 118 & -12 & -14 & 109 & 0,92 \\
\hline 2 & 116 & -10 & -12 & 105 & 0,91 \\
\hline 3 & 116 & -8 & -10 & 85 & 0,73 \\
\hline 4 & 111 & -6 & -8 & 25 & 0,23 \\
\hline 5 & 114 & -4 & -6 & 26 & 0,23 \\
\hline 6 & 198 & -2 & -4 & 45 & 0,23 \\
\hline 7 & 177 & 0 & -2 & 46 & 0,26 \\
\hline 8 & 198 & 2 & 0 & 30 & 0,15 \\
\hline 9 & 201 & 4 & 2 & 25 & 0,12 \\
\hline 10 & 116 & 6 & 4 & 0 & 0 \\
\hline 11 & 118 & 8 & 6 & 0 & 0 \\
\hline 12 & 114 & 10 & 8 & 0 & 0 \\
\hline 13 & 114 & 12 & 10 & 0 & 0 \\
\hline 14 & 98 & 14 & 12 & 0 & 0 \\
\hline 15 & 92 & 16 & 14 & 0 & 0 \\
\hline
\end{tabular}

La Fig. 12 ilustra el comportamiento del algoritmo clásico de detección de energía en diferentes NSR de señales modulas en bpsk y qpsk.

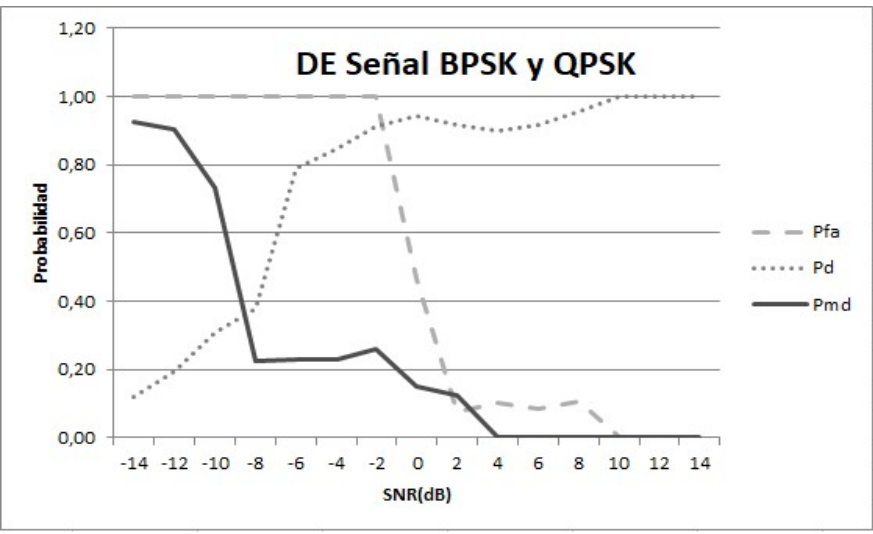

Fig. 12, Probabilidad Pd, Pfa y Pmd de una señal en BPSK y QPSK aplicando técnica de detección de energía. 


\section{CONCLUSIONES Y FUTUROS TRABAJOS}

El algoritmo IZMA sin aplicar la SD, ejecuta muy pocas aproximaciones, con un error medio cuadrático cercano a cero, pero sin recuperar la señal original.

Al comparar las cuatro señales simuladas se observa que la diferencia entre la norma nuclear inicial y la norma nuclear final es mayor para SNR inferiores a $4 \mathrm{~dB}$, es decir que el algoritmo IZMA_SD recupera las muestras de la señal en buenas condiciones de canal.

Un aspecto importante en la ejecución del algoritmo de IZMA_SD es el tiempo que tarda en generar la matriz aproximada, se requieren más de 300 aproximaciones en SNR inferiores a $-8 \mathrm{~dB}$ y esta se reduce a 250 aproximaciones en SNR superiores a $8 \mathrm{~dB}$, nuevamente el algoritmo presenta mayor velocidad al recuperar muestras perdidas en buenas condiciones de canal. La ejecución del algoritmo requiere un recurso máquina considerable y este se puede reducir si se reduce el error medio cuadrático.

Una vez es recuperada la señal por IZMA_SD, se aplica el SS con DE, el cual presenta fallas al detectar un PU cuando existe una SNR muy baja, este problema se puede solucionar si se determina de forma apropiada el valor del umbral de ruido; por lo anterior DE presenta un buen comportamiento cuando las SNR es superior a $2 \mathrm{~dB}$.

En futuros trabajos para evaluar el algoritmo MC IZMA_SD se recomienda implementarlo en una USRP con buenas características de procesamiento, muestreo y resolución del convertidor a/d. Además, se deben implementar con el algoritmo IZMA_SD otras técnicas de SS en banda estrecha como el cicloestacionario, filtro adaptativo, redes neuronales y lógica fuzzy para la detección eficiente de huecos espectrales orientados a banda ancha.

\section{REFERENCIAS}

[1] Cisco. "White Paper: Cisco Visual Networking index: Forecast and Methodology, 2015-2010". Disponible en: http://www.cisco.com/c/en/us/solutions/collateral/serviceprovider/visual-networking-index-vni/complete-white-paper-c11481360.pdf. San José, California, USA, 06/07/2016.

[2] Locke, G., and Strickling, L. E. "Plan and timetable to make available 500 Megahertz of spectrum for wireless broadband." US Department of Commerce, Washington, DC, USA (2010).

[3] FCC Spectrum Policy Task Force (SPTF), "Report of the Spectrum Efficiency Working Group”, United States, November 2002.

[4] Shared Spectrum Company, "General Survey of Radio Frequency Bands - $30 \mathrm{MHz}$ to $3 \mathrm{GHz}$ ", Vienna, Virginia, United States, September 2010.

[5] Rory, V. "A Framework for Radio Frequency Spectrum Measurement and Analysis", Technical Report, the University of Kansas, United States, March 2008

[6] Mitola, J., Maguire, G. Q. “Cognitive Radio making software radios more personal", IEEE Personal Communicant, Vol 6, No. 4, pag. 13-18, September 1999.

[7] Mitola, J. "Cognitive Radio an Integrated Agent Architecture for Software Defined Radio", Dissertation Doctor Technology, Royal Institute of Technology (KTH), Sweden, ISSN 1403-5286, May 2000.

[8] Haykin, S. "Cognitive Radio: Brain-Empowered Wireless Communications", IEEE Journal On Selected Areas In Communications, Vol. 23, No. 2, February 2005.

[9] Aguila, J. H. "Radio Cognitiva- Estado del arte", Universidad Icesi, Revista Sistemas y telemática, vol. 9, No.16, 2011.
[10] NTIA, "United States Frequency Allocation Chart", [online] https://www.ntia.doc.gov/files/ntia/publications/spectrum_wall_chart_a ug2011.pdf

[11] Pawełczak, P. "Cognitive Radio: Ten Years of Experimentation and Development," IEEE Communications Magazine, vol. 49, no. 3, pag. 90100, Mar. 2011.

[12] Peha, J. "Sharing Spectrum Through Spectrum Policy Reform and Cognitive Radio," Proceedings of the IEEE, vol. 97, pag. 708-719, April 2009.

[13] Subhedar, M., y Birajdar G.” Spectrum Sensing Techniques In Cognitive Radio Networks: A Survey", International Journal of Next Generation Networks, vol. 3, No. 2, June 2011.

[14] Christopher, J., Krishnan, V., y Bagubali, A. "Cognitive Radio: Spectrum Sensing Problems in Signal Processing", International Journal of computer applications, vol. 40, No. 16, February 2012.

[15] Rozeha, A., and Norsheila, F."Issues Of Spectrum Sensing In Cognitive Radio Based Systems", Available: http://trg.fke.utm.my/members/rozeha/2_09.pdf Telematic and Optic Department, Faculty of Electrical Engineering, University Technology Malaysia.2010.

[16] Tevfik, Y., and Huseyin, A. "A Survey of Spectrum Sensing Algorithms for Cognitive Radio Applications", IEEE Communications Surveys \& Tutorials, Vol. 11, No. 1, Pag. 116-130, First Quarter 2009.

[17] Hongjian, S., et al. "Wideband spectrum sensing for cognitive radio networks: a survey." IEEE Wireless Communications 20.2 (2013): 7481.

[18] Zhi, Q., et al. "Optimal multiband joint detection for spectrum sensing in cognitive radio networks." IEEE Transactions on Signal Processing 57.3 (2009): 1128-1140.

[19] Hong, H., et al. "Applications of compressed sensing in communications networks." arXiv preprint arXiv:1305.3002 (2013).

[20] Candes, E. J., and Recht B. "Exact Matrix completion via convex optimization. Foundations of Computational mathematics, 9(6), 717.

[21] Shabat, G., \& Averbuch, A. Interest zone matrix approximation. Electronic Journal of Linear Algebra, 23(1), 50 (2012)

[22] Waleed, E." Spectrum Sensing in Cognitive Radio Networks". Tesis de maestría, faculty of Computer Engineering Department College of Electrical and Mechanical Engineering, National University of Sciences and Technology, Pakistan, 2008

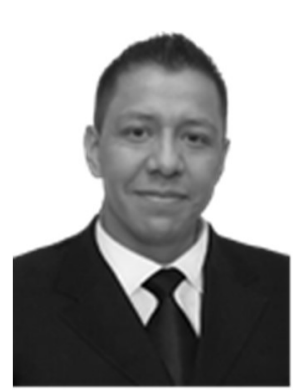

Olger Ferledy Erazo De La Cruz. Ingeniero de sistemas de la Universidad Mariana, Pasto, Colombia (1999). Especialista en redes y servicios telemáticos, Universidad del Cauca, Popayán, Colombia (2001). Especialista en administración de la informática educativa, Universidad de Santander, Bucaramanga, Colombia (2013). Estudiante de maestría en electrónica y telecomunicaciones, Universidad del Cauca, Popayán, Colombia. Sus líneas de interés son: la telemetría y energías renovables. Actualmente es profesor en la Facultad de ingeniería de la Universidad CESMAG, Pasto, Colombia. Miembro del grupo de investigación RAMPA de la Universidad CESMAG. ORCID: https://orcid.org/0000-0003-2995-648X.

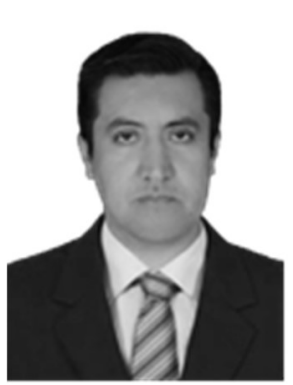

Víctor Fabián Miramá Pérez. Ingeniero en electrónica y telecomunicaciones, Universidad del Cauca, Popayán, Colombia (2008). Magister en electrónica y telecomunicaciones, Universidad del Cauca, Popayán, Colombia (2013). Sus líneas de interés son: gestión del recurso radio, teoría de juegos y redes de próxima generación. Actualmente es profesor en la Facultad de ingeniería de la universidad del Cauca, Popayán, Colombia. Miembro del Grupo de I+D Nuevas Tecnologías en Telecomunicaciones GNTT y grupo de radio e inalámbricas GRIAL de la Universidad del Cauca. ORCID: https://orcid.org/0000-0003-3845-1985. 


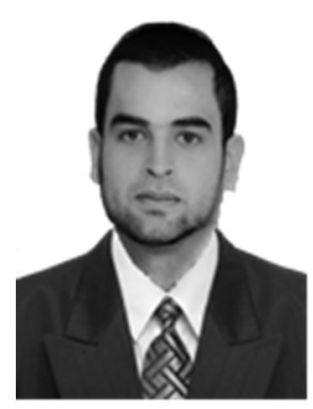

Jorge Edison Mora Arroyo. Ingeniero electrónico de la Universidad de Nariño, Pasto, Colombia (2009). Ingeniero de telecomunicaciones, Universidad Nacional Abierta y a Distancia, Colombia (2018). Estudiante de maestría en electrónica y telecomunicaciones, Universidad del Cauca, Popayán, Colombia. Sus líneas de interés son: redes inalámbricas avanzadas, inteligencia artificial, teoría de juegos y redes heterogéneas. Actualmente trabaja en el Servicio Geológico Colombiano, Pasto.

ORCID: https://orcid.org/0000-0003-3850-4506. 